\title{
Diagnostic Value of Serum Baseline Tryptase Levels in Childhood Asthma and Its Correlation with Disease Severity
}

\author{
Siju Gao Jingang Fan Zhaoan Wang \\ Department of Pediatrics, Linyi People's Hospital, Linyi City, China
}

\author{
Keywords \\ Tryptase · Childhood asthma - Mast cells · Allergy · \\ Pulmonary disease
}

\begin{abstract}
Background: The aim of this study was to explore whether serum baseline tryptase (sBT) levels might be a useful marker not only for the accurate diagnosis of childhood asthma, but also for the prediction of disease severity. Methods: A total of 114 asthmatic children were enrolled in this study, 36 of whom had mild intermittent asthma, 38 had mild persistent asthma, and 40 had moderate to severe persistent asthma. Additionally, 34 age-matched healthy children were enrolled as controls. The sBT levels of these populations were measured using a fluoroenzymeimmunoassay kit. The diagnostic performance of sBT levels and their correlation with asthma severity were systematically investigated using receiver operating characteristic (ROC) analysis and correlation analysis. Results: Children with mild and moderate to severe persistent asthma had significantly increased sBT levels as compared to those with mild intermittent asthma and healthy controls. ROC analysis further demonstrated that SBT levels not only appear to be highly sensitive and specific for distinguishing asthmatic children from
\end{abstract}

\section{KARGER}

(c) 2017 S. Karger AG, Basel

E-Mail karger@karger.com

www.karger.com/iaa healthy controls, but also show good accuracy for the differentiation of various asthmatic subgroups. Correlation analysis revealed that in all asthmatic subgroups sBT levels were significantly correlated with a variety of key markers that reflect the disease severity of asthma, including childhood asthma control test scores, serum IgE and interleukin-13 levels, blood eosinophil counts, and pulmonary test parameters. Conclusions: SBT levels may have a potential use in supporting a diagnosis of asthma in children and as a predictor of disease severity.

(c) 2017 S. Karger AG, Basel

\section{Introduction}

In recent years, multiple approaches have been developed for the diagnosis of asthma, of which pulmonary function tests (spirometry and plethysmography) performed over time are considered to be key [1]. In clinical practice, however, not all children are able to perform pulmonary function tests, and children less than 6 years of age may be unable to produce clinically useful information [1]. Therefore, it is particularly difficult to diagnose asthma in preschool pediatric patients, which creates a great need for novel and accurate diagnostic markers that 
can effectively identify asthmatic children and sufficiently reflect the severity of the disease.

Numerous studies have demonstrated that the pathophysiological mechanisms involved in asthma are heterogenous and complex, and involve numerous cell types, including basophils, macrophages, eosinophils, and mast cells [2]. Mast cells, known for their role in the allergic reaction and anaphylaxis, are critical effectors of asthma [3]. When stimulated by inhaled antigens, sensitized mast cells can be activated to release several vasoactive/spasmogenic mediators (e.g. histamine, cysteinyl leukotrienes, prostaglandin D2, and platelet-activating factor), which induce mucous cell metaplasia and affect airway smooth muscle cells, consequently resulting in narrowing of the airways [2].

Tryptase is a mast cell-specific serine proteinase, which has been documented as a causative factor in multiple allergic and inflammatory disorders $[4,5]$. Recently, accumulating evidence has highlighted the association between serum baseline tryptase (sBT) levels and the severity of several allergic and inflammatory diseases. For example, children with insect venom hypersensitivity were found to have elevated sBT levels, which could be regarded as a strong risk indicator for severe systemic reactions [6]. Another clinical study reported that sBT levels were significantly increased in children with food allergies who experienced anaphylaxis, thereby indicating that sBT levels might also be a good predictor for risk of anaphylaxis [7]. Based on these findings, we hypothesized that sBT might be a useful marker not only for the accurate diagnosis of pediatric patients with asthma, but also for prediction of the disease activity in this population. To test this hypothesis, we performed an observational study in which sBT levels were measured in asthmatic children and healthy controls. The diagnostic performance of sBT levels and their correlation with asthma severity were systematically investigated.

\section{Patients and Methods}

Patients and Healthy Controls

From September 2013 to August 2015, we consecutively recruited asthmatic children from the Department of Pediatrics of the corresponding author's institution as members of a test group. In order to limit potential bias, strict inclusion and exclusion criteria were established prior to recruitment. The inclusion criteria were: (1) age between 5 and 12 years; (2) having mild intermittent or persistent asthma based on the revised Global Initiative for Asthma (GINA) criteria [8], and (3) a willingness to participate in the study expressed by the patient's parents or guardians. The exclusion criteria were: (1) age younger than 5 or older than 12 years; (2) having a pulmonary disorder other than asthma (e.g. cystic fibrosis, chronic bronchiectasis, bronchitis, chronic obstructive pulmonary disease); (3) having a significant medical illness (e.g. malignant disease, severe heart or endocrine disease, severe infectious disease) other than asthma; (4) unable to coordinate use of the spirometric device used in this study (based on the investigator's opinion), and (5) experiencing an asthmatic or allergic exacerbation and therefore receiving systemic corticosteroids or antihistamine within 4 weeks prior to enrollment. In addition, 34 consecutive age-matched healthy children were recruited from health check-ups as controls (the control group) for the study period. At enrollment, a full medical history and physical examination was undertaken in these children to confirm that they had no medical condition or treatment that could affect the sBT level. The study protocol was approved by the ethics committee of the corresponding author's institution. Written informed consent was obtained from the parents and guardians of all participants.

\section{Measurement of sBT Levels}

For all asthmatic children and healthy controls, blood samples were collected at the same time for 3 consecutive days. After centrifugation, $\mathrm{sBT}$ levels were measured using a commercialized fluoroenzymeimmunoassay kit (ImmunoCAP ${ }^{\circledR}$ Tryptase, Thermo Fisher Scientific Inc., Waltham, MA, USA) according to the manufacturer's instructions. For each sample, the assay was performed in duplicate. All measurements were carried out by an experienced physician who was blinded to any clinical information. The average value across all samples was calculated for each subject for final analysis.

\section{Assessment of the Severity of Asthma}

In order to assess the severity of asthma, asthma serum marker measurements were taken, and childhood asthma control tests (CACT), GINA guideline-based severity evaluations, and pulmonary function tests were performed for all asthmatic participants. Briefly, a 7-item questionnaire was used to generate C-ACT scores for asthma control [9], while the revised GINA criteria (published in 2002) were used for severity classification [8]. Pulmonary function tests were performed using a total computerized spirometer (MasterScreen $^{\mathrm{TM}}$, Jaeger Corporation, Höchberg, Germany) according to the standards of the American Thoracic Society (ATS). Prior to testing, all subjects were trained in how to use the spirometer. All tests were carried out between 8:00 a.m. and 4:00 p.m. and replicated 3 times in each subject. The highest levels for forced expiratory volume in $1 \mathrm{~s}\left(\mathrm{FEV}_{1}\right)$, forced vital capacity $(\mathrm{FVC})$, maximal mid-expiratory flow, and peak expiratory flow were taken independently from the 3 curves.

Asthma serum marker measurements included serum levels of total IgE, interleukin-13 (IL-13), and interferon-gamma (IFN- $\gamma$ ), and were examined using corresponding enzyme-linked immunosorbent assay kits (Jiancheng Biotech, Nanjing, China). Assays were performed in duplicate in exact accordance with the manufacturer's instructions. The average values of blood samples collected from each participant were calculated and used for the final analysis. Additionally, blood eosinophil and neutrophil counts were determined by an automated hematology analyzer (ADVIA 120, Bayer Health Care, Berlin, Germany). All measurements were carried out by an experienced physician who was blinded to any clinical information. 
Table 1. Demographic and clinical characteristics of the participants enrolled in this study

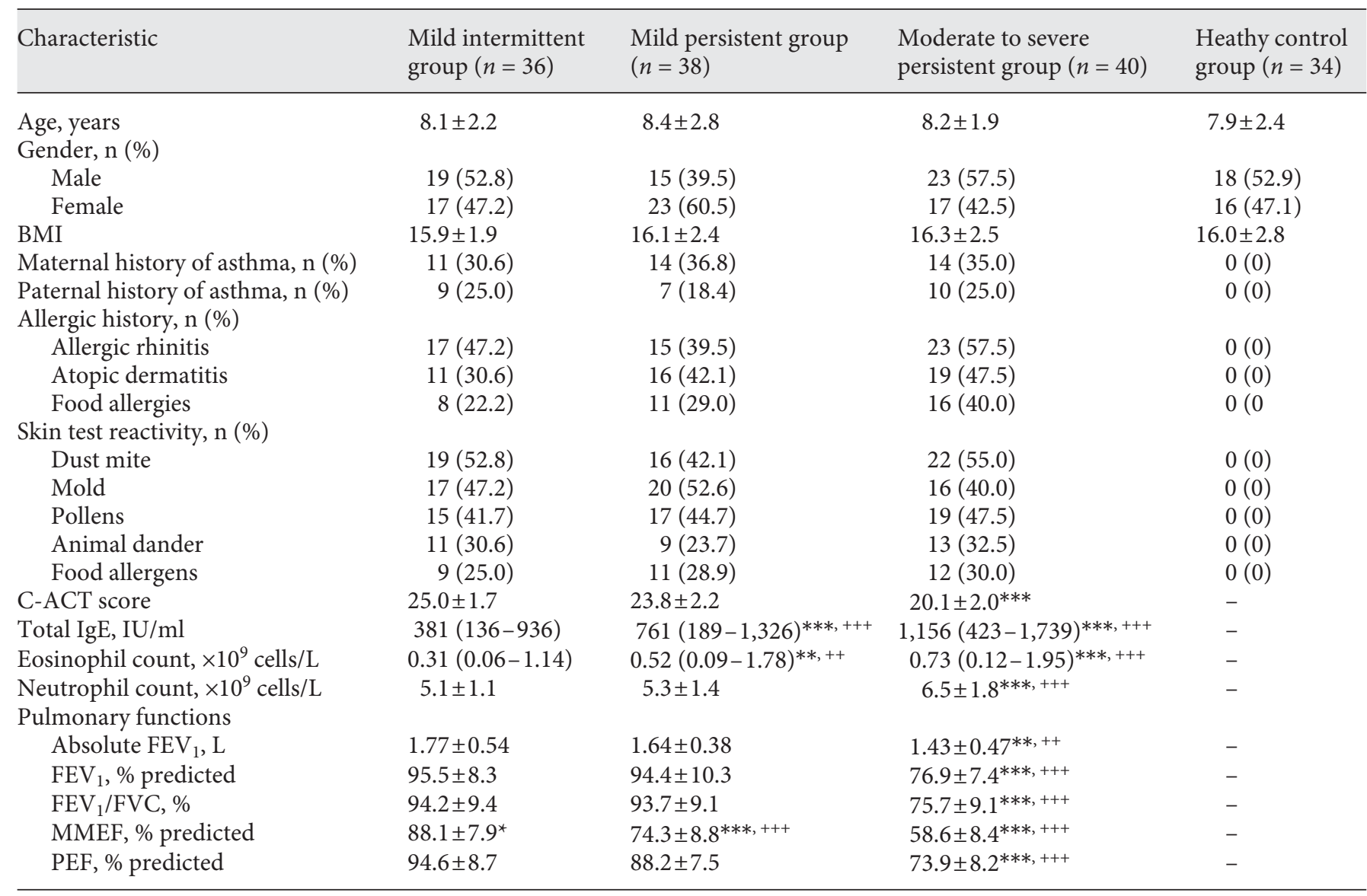

Continuous data with a normal distribution are presented as means $\pm \mathrm{SD}$, while continuous data with a skewed distribution are presented as medians (ranges). BMI, body mass index; C-ACT, childhood asthma control test; IgE, immunoglobulin E; FEV 1 , forced expiratory volume in $1 \mathrm{~s}$; FVC, forced vital capacity; MMEF, maximal mid-expiratory flow; PEF, peak expiratory flow. ${ }^{* *} p<0.01,{ }^{* * *} p<$ 0.001 versus healthy control group; ${ }^{++} p<0.01,{ }^{+++} p<0.001$ versus the mild intermittent group.

\section{Statistical Analysis}

Statistical analysis was conducted using SPSS software (version 19.0, SPSS Inc., Chicago, IL, USA). For continuous data, the Kolmogorov-Smirnov test was used to assess the normality of the distribution. Data that follow a normal distribution are presented as means \pm standard deviation (SD), while data that have a skewed distribution are presented as medians (ranges). Categorical data are expressed as counts and percentages. For continuous data with a normal distribution, 1-way analysis of variance followed by the Tukey post hoc test was used to compare differences among the groups. For continuous data with a skewed distribution, the Kruskal-Wallis test followed by the Dunn post hoc test was used to compare differences among the groups. Statistical comparisons between categorical data were determined using the $\chi^{2}$ test. Receiver operating characteristic (ROC) analysis was used to evaluate the diagnostic accuracy of sBT levels. Pearson and Spearman correlation tests were used for correlation analyses. A $p$ value $<0.05$ was considered statistically significant (2-tailed).

\section{Results}

\section{Demographic and Clinical Characteristics of Participants}

From September 2013 to August 2015, a total of 114 asthmatic children who met the inclusion criteria were enrolled in the current study, $36(31.6 \%)$ of whom had mild intermittent asthma, 38 (33.3\%) had mild persistent asthma, and 40 (35.1\%) had moderate to severe persistent asthma. Of these children, $96(84.2 \%)$ were recruited from outpatient clinics and 18 (15.8\%) were from inpatient facilities. In addition, 34 healthy children were included as controls. Most of the children with moderate to severe persistent asthma had been using inhaled corticosteroids as a treatment, and were allowed to continue 


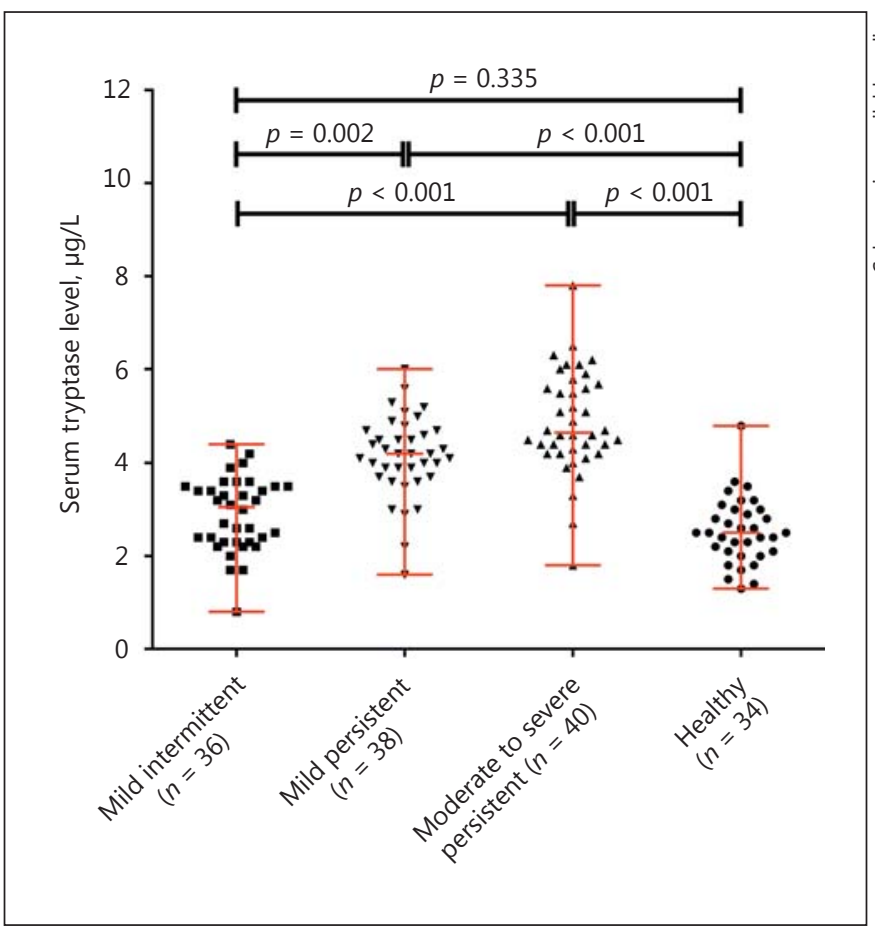

Fig. 1. Comparisons of sBT levels among healthy controls and children with mild intermittent, mild persistent, or moderate to severe persistent asthma. Each dot represents the average value of blood samples collected at the same time for 3 consecutive days. Bars indicate medians and ranges. Statistical differences between the 2 groups were evaluated using the Kruskal-Wallis test followed by the Dunn post hoc test.

their usual treatments during the study period. The demographic and clinical characteristics of all participants are summarized in Table 1 . There were no statistically significant differences in age, gender, or BMI among all asthmatic subgroups and the healthy control group. Furthermore, clinical features including parental asthmatic history, personal allergic history, and skin test reactivity were comparable among the 3 groups of asthmatic cohorts. However, children with moderate to severe persistent asthma had considerably decreased C-ACT scores, as well as significantly increased serum IgE levels and eosinophil and neutrophil counts, as compared to healthy controls and children with mild intermittent asthma $(p<$ 0.001). In addition, the moderate to severe persistent asthma group had the poorest pulmonary function among all study groups.

\section{sBT Levels in Asthmatic Children}

Measurements of sBT levels were available for all of the participants. The results are shown in Figure 1. The me- dian serum sBT levels of the mild persistent and moderate to severe persistent asthma groups were $4.2 \mu \mathrm{g} / \mathrm{L}$ (range 1.6-6.0) and $4.7 \mu \mathrm{g} / \mathrm{L}$ (range 1.8-7.8), respectively, which were significantly greater than those of either the mild intermittent group or the healthy control group. However, the latter 2 groups had similar median serum sBT levels and there was no difference in statistical significance $(3.1 \mu \mathrm{g} / \mathrm{L}$ in the mild intermittent group vs. $2.5 \mu \mathrm{g} / \mathrm{L}$ in the healthy control group, $p=0.335$ ).

\section{Diagnostic Accuracy of sBT Levels in Discriminating Asthmatic Children}

The ROC curve for the sBT level used in discriminating asthmatic children from healthy controls is presented in Figure 2a. The cut-off value was $3.2 \mu \mathrm{g} / \mathrm{L}$, which gave a sensitivity of $75.4 \%$ and a specificity of $88.2 \%$, as well as an area under the curve (AUC) of 0.844 (95\% CI $0.775-$ 0.898 ). These results suggest that sBT levels have a fair to good performance in discriminating asthmatic children from healthy controls. For the sBT level used in diagnosing persistent patients from intermittent patients, the cutoff value was $3.6 \mu \mathrm{g} / \mathrm{L}$ with $85.9 \%$ sensitivity and $88.9 \%$ specificity, and an AUC of 0.906 (95\% CI 0.837-0.953; fig. 2b), indicating good accuracy for such a diagnosis. Furthermore, we investigated the diagnostic accuracy of sBT levels for the different asthmatic subgroups. The corresponding diagnostic parameters are summarized in Table 2. It should be noted that sBT levels showed good diagnostic accuracy for various patient subgroups, except that a relatively weak performance was seen in discriminating patients with mild intermittent asthma from healthy controls (sensitivity $41.7 \%$, specificity $88.2 \%$, AUC $0.646,95 \%$ CI $0.523-0.757$ ). These results can be explained by the fact that there was a significant difference in sBT levels between the intermittent and persistent asthmatics, but this was not the case for mild intermittent asthmatics versus healthy controls.

\section{Correlation between sBT Levels and Disease Activity in Asthmatic Children}

It is well known that IL-13 and IFN- $\gamma$ are important cytokines involved in the pathogenesis of asthma; IL-13 in particular is considered one of the biomarkers for evaluating asthma control, and its serum concentrations correlate with the severity of the disease $[10,11]$. We therefore measured the serum levels of both cytokines across all study groups. As shown in Figure 3a, all asthmatic subgroups showed significantly increased serum IL-13 levels as compared to the healthy control group $(p<0.001$ for each asthmatic subgroup vs. the healthy control group). 


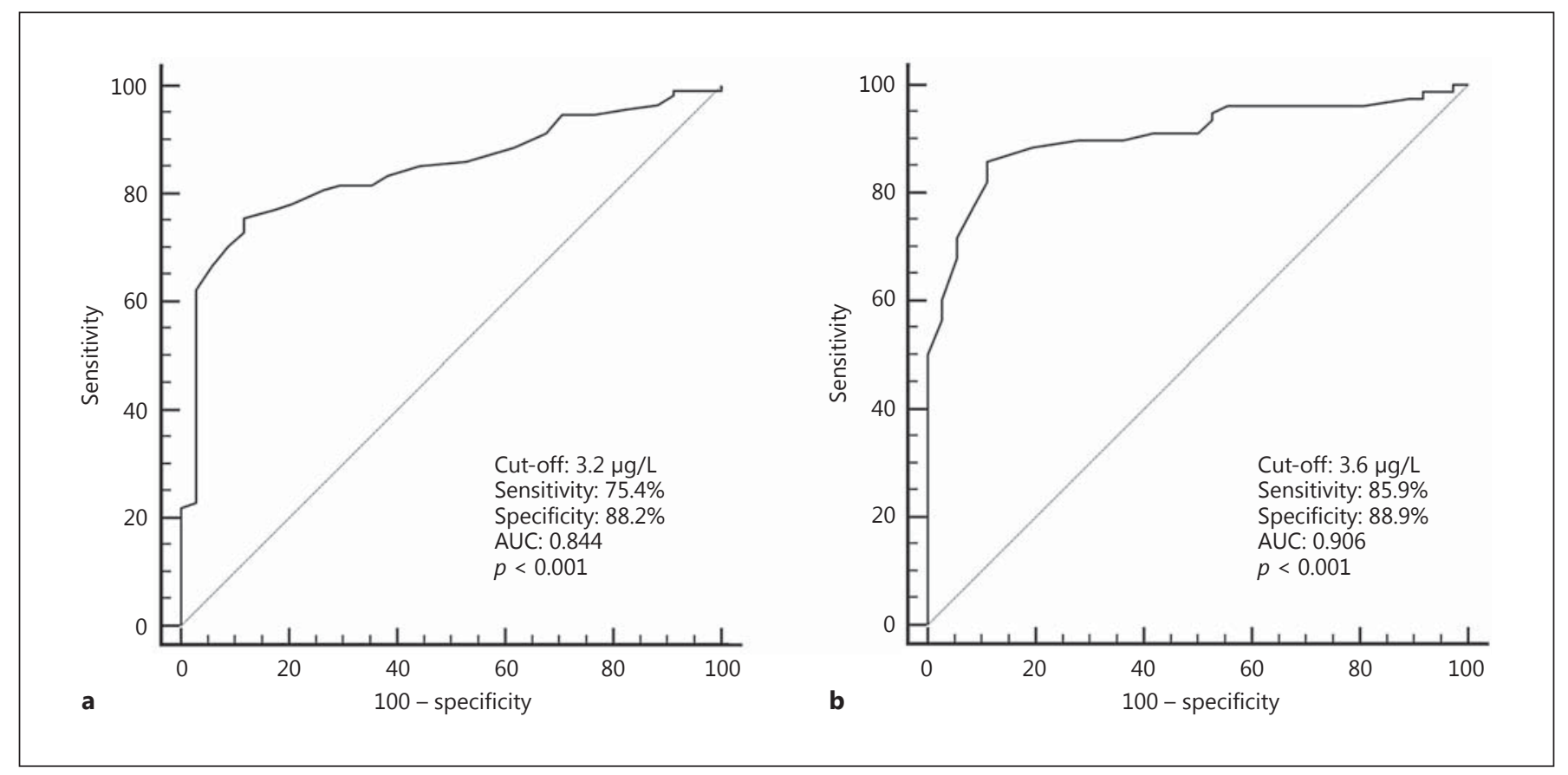

Fig. 2. ROC curves of sBT levels in discriminating asthmatic children from healthy controls (a), and persistent patients from intermittent patients (b). Sensitivity is plotted against $100 \%$ specificity. The cut-off value presented in each panel was determined by ROC analysis. The AUC was calculated to summarize the diagnostic accuracy of each ROC curve. An AUC value $<0.7$ indicates low diagnostic accuracy, $0.7-0.9$ indicates moderate accuracy, and $>0.9$ indicates high accuracy.

Table 2. Diagnostic accuracy of sBT levels for different asthmatic subgroups

\begin{tabular}{|c|c|c|c|c|c|c|}
\hline $\begin{array}{l}\text { Diagnostic } \\
\text { parameters }\end{array}$ & $\begin{array}{l}\text { Mild intermittent } \\
\text { vs. control }\end{array}$ & $\begin{array}{l}\text { Mild persistent } \\
\text { vs. control }\end{array}$ & $\begin{array}{l}\text { Moderate to } \\
\text { severe persistent } \\
\text { vs. control }\end{array}$ & $\begin{array}{l}\text { Mild intermittent } \\
\text { vs. mild persistent }\end{array}$ & $\begin{array}{l}\text { Mild intermittent } \\
\text { vs. moderate to } \\
\text { severe persistent }\end{array}$ & $\begin{array}{l}\text { Mild persistent } \\
\text { vs. moderate to } \\
\text { severe persistent }\end{array}$ \\
\hline Cut-off, $\mu \mathrm{g} / \mathrm{L}$ & 3.2 & 3.5 & 3.6 & 3.6 & 3.6 & 4.3 \\
\hline $\mathrm{TP}, n$ & 15 & 32 & 37 & 30 & 37 & 29 \\
\hline $\mathrm{FP}, n$ & 4 & 2 & 1 & 4 & 4 & 15 \\
\hline $\mathrm{TN}, n$ & 30 & 32 & 33 & 32 & 32 & 23 \\
\hline $\mathrm{FN}, n$ & 21 & 6 & 3 & 8 & 3 & 11 \\
\hline SEN, \% & 41.7 & 84.2 & 92.5 & 78.9 & 92.5 & 72.5 \\
\hline SPE, $\%$ & 88.2 & 94.1 & 97.1 & 88.9 & 88.9 & 60.5 \\
\hline PPV,$\%$ & 78.9 & 94.1 & 97.4 & 88.2 & 90.2 & 65.9 \\
\hline NPV, \% & 58.8 & 84.2 & 91.7 & 80.0 & 91.4 & 67.6 \\
\hline PLR & 3.6 & 14.3 & 31.5 & 7.1 & 8.3 & 1.8 \\
\hline NLR & 0.66 & 0.17 & 0.08 & 0.24 & 0.08 & 0.45 \\
\hline $\operatorname{AUC}(95 \% \mathrm{CI})$ & $0.646(0.523-0.757)$ & $0.916(0.827-0.969)$ & $0.953(0.876-0.988)$ & $0.870(0.771-0.937)$ & $0.940(0.861-0.982)$ & $0.711(0.597-0.808)$ \\
\hline$p$ value & 0.028 & $<0.001$ & $<0.001$ & $<0.001$ & $<0.001$ & $<0.001$ \\
\hline
\end{tabular}

TP, true positive; FP, false positive; TN, true negative; FN, false negative; SEN, sensitivity; SPE, specificity; PPV, positive predictive value; NPV, negative predictive value; PLR, positive likelihood ratio; NLR, negative likelihood ratio, AUC, area under the curve.

Children with moderate to severe persistent asthma had the highest median serum levels $(49.7 \mu \mathrm{g} / \mathrm{L}$, range $22.2-$ 59.3). For serum IFN- $\gamma$ levels, however, we observed a considerable decrease in the mild and moderate to severe persistent groups as compared with the mild intermittent and healthy control groups, and children with moderate to severe persistent asthma had the lowest median serum levels $(39.4 \mu \mathrm{g} / \mathrm{L}$, range 25.1-56.4; Fig. 3b). The ratio of IFN- $\gamma$ to IL-13 was calculated for each study group based on the measured levels of IL-13 and IFN- $\gamma$. As shown in Figure $3 c$, the ratio of IFN- $\gamma$ to IL- 13 was significantly lower in the 3 asthmatic subgroups than in the healthy 

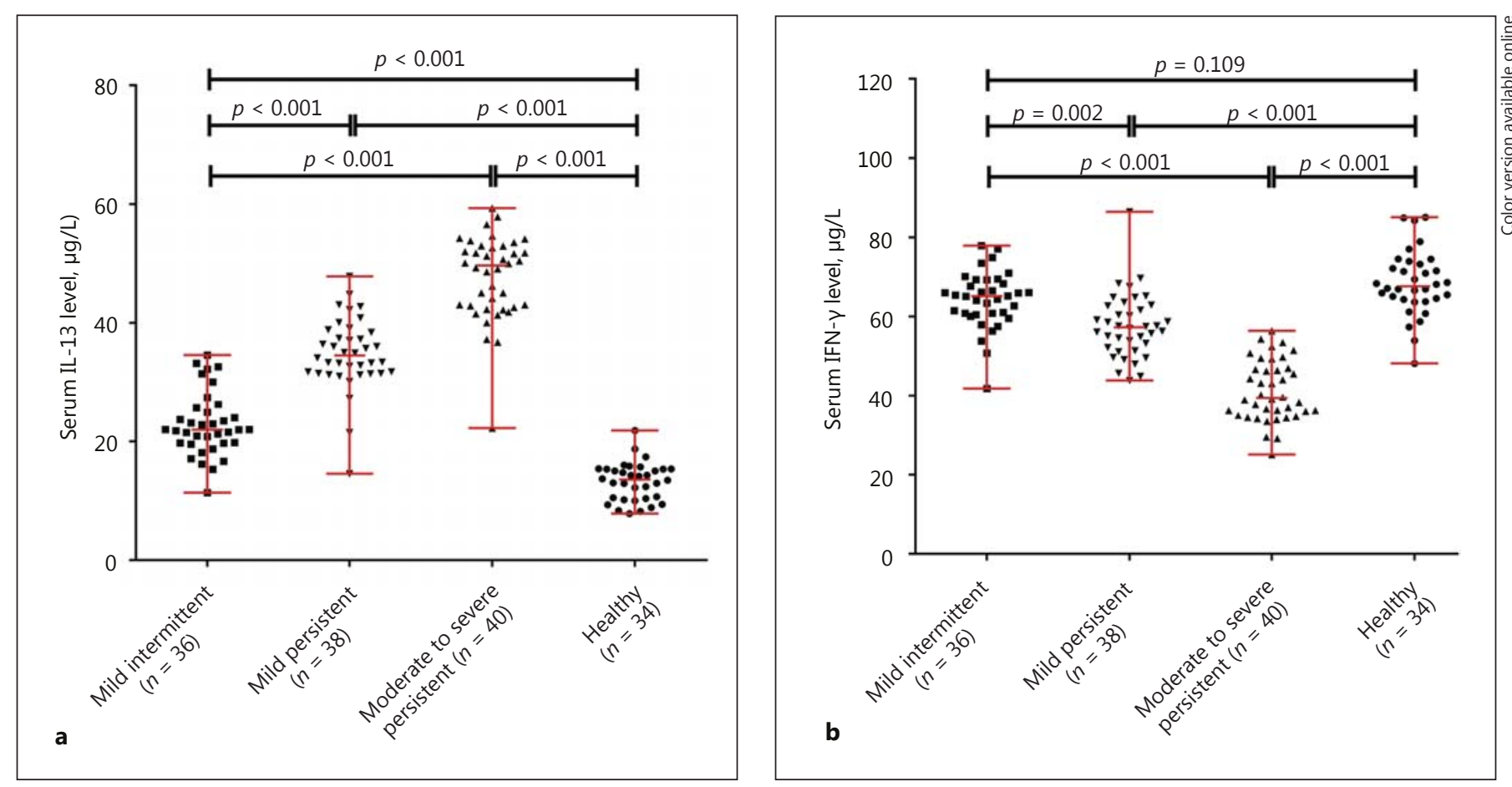

Fig. 3. Comparisons of serum IL-13 (a) and IFN- $\gamma$ levels (b), and the ratio of IFN- $\gamma$ to IL-13 (c) among healthy controls and children with mild intermittent, mild persistent, or moderate to severe persistent asthma. Each dot represents the average value of blood samples collected at the same time for 3 consecutive days. Bars indicate medians and ranges. Statistical differences between the 2 groups were evaluated using the Kruskal-Wallis test followed by the Dunn post hoc test.

control group, and showed a negative relationship with the increasing severity of asthma.

Next, we explored the correlation between sBT levels and disease severity in asthmatic children. As shown in Table 3, sBT levels showed a strong correlation with C-

ACT scores, serum IgE levels, and eosinophil counts in all asthmatic subgroups; however, no significant correlation was found between C-ACT scores and sBT levels in the mild intermittent group. Additionally, we found that the parameters reflecting pulmonary function (including 
Table 3. Correlation between sBT levels and disease severity in asthmatic children

\begin{tabular}{|c|c|c|c|c|c|c|}
\hline \multirow[t]{2}{*}{ Parameter } & \multicolumn{2}{|c|}{ Mild intermittent group $(n=36)$} & \multicolumn{2}{|c|}{ Mild persistent group $(n=38)$} & \multicolumn{2}{|c|}{$\begin{array}{l}\text { Moderate to severe persistent group } \\
(n=40)\end{array}$} \\
\hline & $r(95 \% \mathrm{CI})$ & $p$ value & $r(95 \% \mathrm{CI})$ & $p$ value & $r(95 \% \mathrm{CI})$ & $p$ value \\
\hline C-ACT score & $-0.105(-0.419$ to 0.232$)$ & 0.544 & $-0.353(-0.605$ to -0.038$)$ & 0.030 & $-0.476(-0.686$ to -0.193$)$ & 0.002 \\
\hline Total IgE, IU/ml & 0.727 (0.523 to 0.852$)$ & $<0.001$ & $0.366(0.053$ to 0.614$)$ & 0.024 & $0.537(0.270$ to 0.727$)$ & $<0.001$ \\
\hline Eosinophil count, $\times 10^{9}$ cells $/ \mathrm{L}$ & $0.716(0.507$ to 0.846$)$ & $<0.001$ & 0.487 (0.198 to 0.698$)$ & 0.002 & $0.440(0.149$ to 0.661$)$ & 0.005 \\
\hline $\mathrm{FEV}_{1}, \%$ predicted & $-0.566(-0.754$ to -0.291$)$ & $<0.001$ & $-0.356(-0.606$ to -0.040$)$ & 0.029 & $-0.498(-0.701$ to -0.221$)$ & 0.001 \\
\hline $\mathrm{FEV}_{1} / \mathrm{FVC}, \%$ & $-0.653(-0.808$ to 0.413$)$ & $<0.001$ & $-0.343(-0.598$ to -0.027$)$ & 0.035 & $-0.455(-0.671$ to -0.167$)$ & 0.003 \\
\hline $\mathrm{IL}-13, \mu \mathrm{g} / \mathrm{L}$ & $0.644(0.400$ to 0.803$)$ & $<0.001$ & $0.492(0.204$ to 0.701$)$ & 0.002 & $0.636(0.405$ to 0.791$)$ & $<0.001$ \\
\hline $\mathrm{IFN}-\gamma, \mu \mathrm{g} / \mathrm{L}$ & $0.136(-0.202-0.445)$ & 0.136 & $0.126(-0.202-0.428)$ & 0.453 & $0.107(-0.212$ to 0.405$)$ & 0.513 \\
\hline Ratio of IFN- $\gamma /$ IL-13 & $-0.601(-0.776$ to -0.339$)$ & $<0.001$ & $-0.479(-0.692$ to -0.188$)$ & 0.002 & $-0.426(-0.651$ to -0.132$)$ & 0.006 \\
\hline
\end{tabular}

C-ACT, childhood asthma control test; IgE, immunoglobulin E; $\mathrm{FEV}_{1}$, forced expiratory volume in $1 \mathrm{~s}$; FVC, forced vital capacity; MMEF, maximal mid-expiratory flow; PEF, peak expiratory flow; IL-13, interleukin-13; IFN- $\gamma$, interferon-gamma.

predicted $\mathrm{FEV}_{1}$ and the ratio of $\mathrm{FEV}_{1}$ to $\mathrm{FVC}$ ), as well as serum IL-13 levels and the ratio of IFN- $\gamma$ to IL-13, were closely associated with sBT levels in various patient subgroups, but this was not the case for serum IFN- $\gamma$ levels.

\section{Discussion}

In this study, we measured sBT levels in 114 asthmatic children and 34 healthy controls, and found that children with mild and moderate to severe persistent asthma had significantly increased sBT levels as compared to those with mild intermittent asthma and healthy controls. ROC analysis further demonstrated that sBT levels not only appear to be highly sensitive and specific for discriminating asthmatic children from healthy controls, but also show good accuracy for the differentiation of various asthmatic subgroups. Correlation analysis revealed that in all asthmatic subgroups, sBT levels were significantly correlated with a variety of key markers that reflect asthma disease severity. These findings suggest that $\mathrm{sBT}$ levels may have great potential for use as a diagnostic indicator of childhood asthma and as a predictor of disease severity.

Tryptase is a predominant protease found in human mast cells, where it resides in secretory granules and can be released in parallel with other mediators (such as histamine and proteoglycans) during mast cell degranulation [12]. As tryptase is released, nearby mast cells are triggered to degranulate and release their contents, thereby attracting more cells to the site of activation and consequently bringing about a rapid amplification of the reaction [13]. Recent studies have revealed that this process is involved in the pathogenesis of a variety of allergic and inflammatory disorders, including allergic rhinitis, venom allergy, psoriasis, and rheumatoid arthritis [14].
Therefore, considering that tryptase is a key marker of mast cell activation, it is plausible to assume that there is an underlying association between sBT levels and the occurrence of these allergic and inflammatory reactions. Researchers have demonstrated that sBT levels are elevated in venom-allergic patients and correlate with the severity of hymenoptera stings [15], while Yavuz et al. [6] reported that elevated sBT levels are a strong risk indicator for severe systemic reactions in children with insect venom hypersensitivity. In addition, Sahiner et al. [7] found that in children with food allergies, sBT concentrations were significantly increased in the population in which anaphylaxis was present, thereby indicating that sBT levels might be a good predictor for risk of anaphylaxis. In line with these findings, our study shows that the children with mild and moderate to severe persistent asthma had remarkably higher sBT levels than healthy controls. Furthermore, sBT levels showed a strong performance in discriminating asthmatic children from healthy controls, as well as in differentiating various asthmatic subgroups. These results indicate the strong association between sBT levels and asthma severity.

Asthma severity is reflected in many aspects of the disease, one of which is bronchial hyperresponsiveness (BHR), an exaggerated response to stimuli which leads to narrowing of the airways $[16,17]$. It has been well documented that BHR is associated with increased numbers of eosinophils and mast cells in the bronchial airway [18]. A previous study also demonstrated that activated mast cells may play an essential role in the development of BHR in children with chronic asthma, which may be dependent on tryptase-induced upregulation of bronchial smooth muscle tone [19]. Hence, it is reasonable to speculate that high sBT levels may reflect the degree of BHR, thus serving as an indirect but reliable indicator of asthma severity. 
This could be supported by the findings of our study, which showed a strong correlation between sBT levels and serum IgE levels, eosinophil counts, and pulmonary function parameters across all asthmatic subgroups. In addition, we found a significant correlation between sBT levels and serum levels of the IL-13 pro-inflammatory cytokine, thereby suggesting that tryptase may also be associated with asthmatic inflammatory responses.

Airway inflammation is another key pathophysiologic feature of asthma which has been demonstrated to be closely linked to disease severity [20]. In the past few decades, numerous studies have identified T helper 2 (Th2) cytokines (such as IL-4, IL-5, and IL-13) as contributors to the airway inflammatory response found in asthma [21]. In addition, Th2 cytokines can be directly released from activated mast cells and subsequently promote Th2 differentiation and an influx of lymphocytes and eosinophils $[22,23]$. Therefore, considering that tryptase is a unique marker of mast cells, sBT levels naturally indicate an inflammatory response, which was reflected in this study by the apparent correlation between sBT levels and serum IL-13 levels. Furthermore, it has been demonstrated that the Th1 cytokine IFN- $\gamma$ acts simultaneously with Th2 cytokines to maintain a chronic inflammatory response in asthmatic airways [23]. In the current study, however, we found that the ratio of IFN- $\gamma$ to IL-13, instead of serum IFN- $\gamma$ levels, was negatively correlated with sBT levels in various asthmatic subgroups. An altered IFN- $\gamma$ to IL-13 ratio usually reflects a Th1/Th2 imbalance, which is generally known to be implicated in the development of asthma [20]. Our data imply that elevated sBT levels may be an indirect warning sign of a Th1/Th2 imbalance in asthmatic children. Nevertheless, further studies are still needed to corroborate this claim.

Several limitations should be addressed regarding this study. First, the small sample size of the study populations may reduce the impact of our findings. Secondly, all participants were from a single ethnicity (Chinese) and were enrolled at a single institution, which may affect the ex- trapolation of the results. Additionally, for the children in the control group, although their full medical history records and skin testing results indicated that they had no medical conditions that could affect sBT level, the lack of data on urine cotinine level, serum IgE level, and pulmonary functions may hamper ascertainment of their passive smoking history, as well as whether they were truly nonatopic and whether they had no suggestion of mild asthma. Furthermore, it is known that most asthmatic children have atopy, while a significant proportion of atopic children do not have asthma [24]. The current study does not include a separate nonasthmatic atopic control group, which is another limitation as it is still unclear whether sBT level can be used as a marker to effectively differentiate nonasthmatic atopic patients from those with mild intermittent asthma. Finally, due to the observational nature of this study, results may be influenced by selection bias and unmeasured confounding, as well as by lack of validation with a blinded test set.

In conclusion, this study demonstrated that sBT levels have a fair to good performance in discriminating between asthmatic children and healthy nonatopic controls, as well as in differentiating various asthmatic subgroups. Moreover, sBT levels are closely associated with a variety of key markers reflecting the severity of asthma. These findings suggest that sBT levels may have a potential use in supporting a diagnosis of asthma in children and a predictor of disease severity.

\section{Acknowledgements}

All authors thank Ms. Rebekah A. Burdyshaw for her kind assistance in proofreading this paper.

\section{Disclosure Statement}

All authors declare that they have no conflicts of interest regarding this study.

\section{References}

Tryptase in the Diagnosis of Childhood Asthma
1 Bush A, Fleming L: Diagnosis and management of asthma in children. BMJ 2015; 350:h996.

2 Barnes PJ: Immunology of asthma and chronic obstructive pulmonary disease. Nat Rev Immunol 2008;8:183-192.

3 Puxeddu I, Levi-Schaffer F: Mast cells and eosinophils: the hallmark of asthma. Paediatr Respir Rev 2004;5(suppl A):S31-S34.
4 Caughey GH: Of mites and men: trypsin-like proteases in the lungs. Am J Respir Cell Mol Biol 1997;16:621-628.

5 Vitte J: Human mast cell tryptase in biology and medicine. Mol Immunol 2015;63:18-24.

6 Yavuz ST, Sackesen C, Sahiner UM, Buyuktiryaki B, Arik Yilmaz E, Sekerel BE, Soyer OU, Tuncer A: Importance of serum basal tryptase levels in children with insect venom allergy. Allergy 2013;68:386-391. 
7 Sahiner UM, Yavuz ST, Buyuktiryaki B, Cavkaytar O, Yilmaz EA, Tuncer A, Sackesen C: Serum basal tryptase may be a good marker for predicting the risk of anaphylaxis in children with food allergy. Allergy 2014;69: 265-268.

8 Hart SP: Asthma severity and adequacy of management. Lancet 2002;359:75.

9 Voorend-van Bergen S, Vaessen-Verberne AA, de Jongste JC, Pijnenburg MW: Asthma control questionnaires in the management of asthma in children: a review. Pediatr Pulmonol 2015;50:202-208.

10 Joseph J, Benedict S, Safa W, Joseph M: Serum interleukin-5 levels are elevated in mild and moderate persistent asthma irrespective of regular inhaled glucocorticoid therapy. BMC Pulm Med 2004;4:2.

11 Gaye B, Sikkema D, Lee TN: Development of an ultra-sensitive single molecule counting assay for the detection of interleukin-13 as a marker for asthmatic severity. J Immunol Methods 2015;426:82-85.
12 Matsson P, Enander I, Andersson AS, Nystrand J, Schwartz L, Watkins J: Evaluation of mast cell activation (tryptase) in two patients suffering from drug-induced hypotensoid reactions. Agents Actions 1991;33:218-220.

13 Amin K: The role of mast cells in allergic inflammation. Respir Med 2012;106:9-14.

14 Frenzel L, Hermine O: Mast cells and inflammation. Joint Bone Spine 2013;80:141-145.

15 Kucharewicz I, Bodzenta-Lukaszyk A, Szymanski W, Mroczko B, Szmitkowski M: Basal serum tryptase level correlates with severity of hymenoptera sting and age. J Investig Allergol Clin Immunol 2007;17:65-69.

16 Pattemore PK, Holgate ST: Bronchial hyperresponsiveness and its relationship to asthma in childhood. Clin Exp Allergy 1993;23:886900.

17 Brannan JD: Bronchial hyperresponsiveness in the assessment of asthma control: airway hyperresponsiveness in asthma: its measurement and clinical significance. Chest 2010; 138:11S-17S.

18 Ediger D, Sin BA, Heper A, Anadolu Y, Misirligil Z: Airway inflammation in nasal polyposis: immunopathological aspects of relation to asthma. Clin Exp Allergy 2005;35:319-326.
19 Ferguson AC, Whitelaw M, Brown H: Correlation of bronchial eosinophil and mast cell activation with bronchial hyperresponsiveness in children with asthma. J Allergy Clin Immunol 1992;90:609-613.

20 Ngoc PL, Gold DR, Tzianabos AO, Weiss ST, Celedon JC: Cytokines, allergy, and asthma. Curr Opin Allergy Clin Immunol 2005;5: 161-166.

21 Holt PG, Sly PD: Th2 cytokines in the asthma late-phase response. Lancet 2007;370:13961398.

22 Barnes PJ: Th2 cytokines and asthma: an introduction. Respir Res 2001;2:64-65.

23 Prescott SL: New concepts of cytokines in asthma: is the Th2/Th1 paradigm out the window? J Paediatr Child Health 2003;39:575579 .

24 Miraglia Del Giudice M, Pedullà M, Piacentini GL, Capristo C, Brunese FP, Decimo F, Maiello N, Capristo AF: Atopy and house dust mite sensitization as risk factors for asthma in children. Allergy 2002;57:169-172. 